\title{
Analysis for temperature and pressure fields in process of hydrate dissociation by depressurization
}

\author{
Zhenwei Zhao ${ }^{1,3}$ and Xinchun Shang ${ }^{2, *, \dagger}$ \\ ${ }^{1}$ Department of Civil Engineering, University of Science and Technology Beijing, Beijing, \\ People's Republic of China \\ ${ }^{2}$ Department of Mathematics and Mechanics, University of Science and Technology Beijing, Beijing, \\ People's Republic of China \\ ${ }^{3}$ Institute of Mechanics, Chinese Academy of Sciences, Beijing, People's Republic of China
}

\begin{abstract}
SUMMARY
An improved axisymmetric mathematic modeling is proposed for the process of hydrate dissociation by depressurization around vertical well. To reckon in the effect of latent heat of gas hydrate at the decomposition front, the energy balance equation is employed. The semi-analytic solutions for temperature and pressure fields are obtained by using Boltzmann-transformation. The location of decomposition front is determined by solving initial value problem for system of ordinary differential equations. The distributions of pressure and temperature along horizontal radiate in the reservoir are calculated. The numeric results indicate that the moving speed of decomposition front is sensitively dependent on the well pressure and the sediment permeability. Copyright (C) 2010 John Wiley \& Sons, Ltd.
\end{abstract}

Received 8 July 2009; Revised 10 November 2009; Accepted 19 November 2009

KEY WORDS: semi-analytic solution; gas hydrate; free boundary value problem; dissociation; depressurization

\section{INTRODUCTION}

Gas hydrate in the permafrost and ocean sediments is a kind of crystalline solid composed of natural gas and hydrogen-bonded water molecules, which is formed under the conditions of higher pressure and lower temperature. It is usually called methane hydrate $\left(\mathrm{CH}_{4} \times\left(\mathrm{H}_{2} \mathrm{O}\right)_{n}\right)$, as the natural gas in geological system involves mainly methane. As a future energy resource available, natural gas trapped in the state of hydrate is estimated as several times to the known conventional natural gas; therefore, developing methods for commercial production of natural gas from hydrates is attracting considerable attention in energy engineering. There are three methods to decompose

\footnotetext{
*Correspondence to: Xinchun Shang, Department of Mathematics and Mechanics, University of Science and Technology Beijing, Beijing, People's Republic of China.

†E-mail: shangxc@ public3.bta.net.cn

Copyright (C) 2010 John Wiley \& Sons, Ltd.
} 
the hydrates: (1) increasing the temperature in geological system over the temperature of hydrate formation and remaining the pressure, (2) decreasing the pressure in the system under the pressure of hydrate formation at a constant temperature, (3) injecting inhibitors to shift the equilibrium of pressure and temperature. Depressurization is generally considered as a favorable method in natural gas production from hydrate.

Based on thermodynamics and continuum mechanics, several mathematic models of the hydrate dissociation process have been reported in the references. The mass and heat transfer for the depressurization process in a hydrate reservoir overlaying a free natural gas zone has been simulated by Holder et al. [1]. An extended model, which considering the effect of water produced by the dissociated hydrate on temperature, has been suggested by Burshears et al. [2]. One-dimensional model for depressurization of natural gas hydrate has been discussed by Yousif and Sloan et al. In this model the process of hydrate dissociation has been assumed to be isothermal, and an analytic solution for pressure has been obtained [3]. Later, the model has been developed by considering region of phase transition, and numerical solution has been obtained by using finite difference [4]. The gas production from hydrate based on the thermal stimulation method has been calculated by Durgut and Parlaktuna [5]. The hydrate formation and decomposition processes have been extensively reviewed by Makogon [6] and Sloan [7]. The mass and energy balance equations at the decomposition front have been introduced to an one-dimensional model, and relative analytic solutions have been obtained by Tsypkin [8]. One-dimensional linearized model was used to find analytic solution by Chuang ji et al. [9] and numerical solution reckoned in the energy balance at the decomposition was subsequently obtained by the finite difference method [10].

Though one-dimensional models have been used to solve analytic and numeric solutions by many authors, and also some of them have considered the energy balance at the decomposition front, axisymmetric model could be a better option. As the actual status of the hydrate dissociation around vertical well is process of horizontal radiating, pressure and temperature fields depend on radial coordinate only. Recently, an axisymmetric linearized model was provided, and a set of approximately analytic solutions for the variations of pressure, temperature and flow rate across the reservoir have been obtained by Ahmadi et al. [11]; however, the effect of latent heat on energy balance at the decomposition front is ignored.

The present investigation intents to analyze temperature and pressure fields in process of hydrate dissociation by depressurization, and natural gas production from methane hydrate. In view of the effect of latent heat of gas hydrate at the decomposition front, an improved axisymmetric model including energy balance equation is suggested by authors. By the means of Boltzmanntransformation, semi-analytic solutions for temperature and pressure fields are obtained. In order to determine unknown functions in the semi-analytic solutions, numeric approach is employed. The numeric results indicate the features of temperature and pressure fields in the process of hydrate dissociation.

\section{MATHEMATIC MODELING}

\subsection{The description of hydrate dissociation process}

The hydrates in the south China sea are contained in porous medium, the porous medium is clay sediment. The porosity of clay layer is 0.4 , the saturation of hydrate is $30 \%$, and the remainder of the pore is water. As the saturation of water reaches $96 \%$ after the hydrate is decomposed fully, 


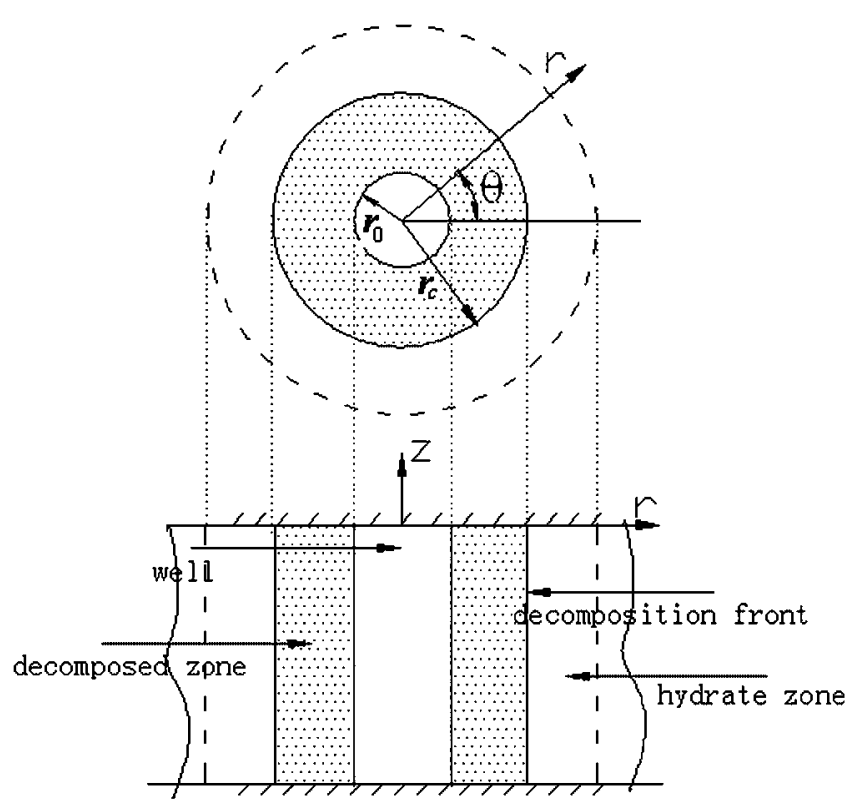

Figure 1. Schematic of hydrates decompose for axisymmetric model.

only the water pressure is considered here, that is, the effect of gas on pressure can be ignored. It is assumed that there is a down-hole pressure well in the reservoir, the pressure of the reservoir decreases while the pressure of the well is dropped, then the hydrates decompose if the pressure is under the balance pressure $P_{\mathrm{D}}$ at a specified temperature. It is assumed that the hydrates decompose quickly when the pressure is under $P_{\mathrm{D}}$. The reservoir is divided into two zones: the decomposed zone and the hydrate zone.

To describe in mathematics, the reservoir is divided into two zones: the decomposed zone and the hydrate zone. In order to describe the pressure and temperature fields in around vertical well for the decomposed zone and the hydrate zone, we introduce the column radial coordinate $(r, \theta, z)$ shown in Figure 1. In view of the actual status of hydrate dissociation, the pressure and temperature fields are assumed to be axisymmetric, that is, they depend on space coordinate $r$ and time $t$ only. The radius of well is denoted as $r_{0}$ and the distance of the decomposition front to the well center as $r=r_{c}(t)$. The decomposition front is interface between the decomposed zone $\left(r_{0}<r<r_{c}\right)$ and the hydrate zone $\left(r_{c}<r<+\infty\right)$. Denote the pressure field as $P_{i}(r, t)$ and the temperature field as $T_{i}(r, t)(i=1,2)$. In this paper, the subscript $i$ identifies the region, with $i=1$ corresponding to the decomposed zone and 2 to the hydrate zone, respectively.

\subsection{The equations of pressure and temperature fields}

On the basis of the mass balance and Darcy's law for flow in an elastic porous medium, pressure distributions in the reservoirs are described by the following equations:

$$
\alpha_{1} \frac{\partial P_{1}}{\partial t}-\frac{1}{r} \frac{\partial}{\partial r}\left(r \frac{\partial P_{1}}{\partial r}\right)=0 \quad\left(r_{0}<r<r_{c}\right)
$$




$$
\alpha_{2} \frac{\partial P_{2}}{\partial t}-\frac{1}{r} \frac{\partial}{\partial r}\left(r \frac{\partial P_{2}}{\partial r}\right)=0 \quad\left(r_{c}<r<+\infty\right)
$$

where the coefficient

$$
\alpha_{i}=\frac{E_{i}}{3\left(1-2 v_{i}\right)} \frac{K_{i}}{\mu} \quad(i=1,2)
$$

$E_{i}$ and $v_{i}$ are Young's modulus and Poisson's ratio of the sediments as elastic porous medium, respectively, $\mu$ the viscosity of water, $K_{i}$ the permeability.

As the conductivity of the sediments porous medium is tiny, the conductive heat transfer in sediments is neglected. The heat is mainly transferred by convection and the influence of the pressure on temperature field should be considered. To describe coupling effect of temperature and pressure, Ahmadi has given the equations of heat convection as follows [9]:

$$
\begin{array}{ll}
\frac{\partial T_{1}}{\partial t}-\frac{c_{\mathrm{W}} k_{1}}{c_{1} \mu} \frac{\partial P_{1}}{\partial r}\left(\frac{\partial T_{1}}{\partial r}-\delta \frac{\partial P_{1}}{\partial r}\right)-\eta \frac{\phi_{1} c_{\mathrm{W}}}{c_{1}} \frac{\partial P_{1}}{\partial t}=0 & \left(r_{0}<r<r_{c}(t)\right) \\
\frac{\partial T_{2}}{\partial t}-\frac{c_{w} k_{2}}{c_{2} \mu} \frac{\partial P_{2}}{\partial r}\left(\frac{\partial T_{2}}{\partial r}-\delta \frac{\partial P_{2}}{\partial r}\right)-\eta \frac{\phi_{2} c_{\mathrm{W}}}{c_{2}} \frac{\partial P_{2}}{\partial t}=0 & \left(r_{c}(t)<r<+\infty\right)
\end{array}
$$

where the constant $c_{\mathrm{w}}$ is the volume heat capacity of water in, $\delta$ is the throttling coefficient, $\eta$ is the adiabatic coefficient of the water, $c_{i}$ is the heat capacity, and $\phi_{i}$ is the porosity of the sediment porous medium $(i=1,2)$.

\subsection{The governing equations at the decomposition front}

In terms of the phase equilibrium for natural gas and hydrate, the temperature and the pressure at the decomposition front $r=r_{c}(t)$ have the relationship [9]

$$
\log _{10} P_{\mathrm{D}}=a_{0}\left(T_{\mathrm{D}}-T_{0}\right)+b_{0}\left(T_{\mathrm{D}}-T_{0}\right)^{2}+c_{0}
$$

where $T_{0}=273.15 \mathrm{~K}^{-1}$, the empirical constants $a_{0}=0.0342 \mathrm{~K}^{-1}, b_{0}=0.0005 \mathrm{~K}^{-2}, c_{0}=6.4804$. $P_{\mathrm{D}}$ and $T_{\mathrm{D}}$ are the pressure and the temperature at the decomposition front, respectively.

Also, the water part produced from the decomposed hydrate should be reckoned in the mass balance equation at the decomposition front, this results in

$$
\chi_{1} \frac{\partial P_{1}}{\partial r}-\chi_{2} \frac{\partial P_{2}}{\partial r}=-\frac{\mathrm{d} r_{c}}{\mathrm{~d} t}
$$

where coefficients

$$
\chi_{i}=\frac{K_{i} \phi_{i}}{\mu\left(\varepsilon \rho / \rho_{w}-\phi_{1}+\phi_{2}\right)} \quad(i=1,2),
$$

$\rho_{w}$ and $\rho$ are, respectively, the density of water and sediment, and $\varepsilon$ is the mass fraction of hydrates in sediments.

Further, when the hydrate is decomposed at the decomposition front, it will absorb heat from surrounding medium. Here, the effect of latent heat is considered, the energy balance equation at 
the decomposition front gives

$$
-\xi_{1} T_{1} \frac{\partial P_{1}}{\partial r}+\xi_{2} T_{2} \frac{\partial P_{2}}{\partial r}=\frac{\mathrm{d} r_{c}}{\mathrm{~d} t}
$$

where coefficients $\xi_{i}=k_{i} \phi_{i} \rho_{w} c_{\mathrm{w}} / \mu L \rho \varepsilon(i=1,2), L$ is the latent heat of hydrate.

\subsection{The conditions to determine solution}

Assume that the pressures and the temperatures in the wall of well and in the infinite are different constants, the boundary conditions are

$$
\begin{aligned}
P_{1}\left(r_{0}, t\right) & =P_{e} \\
P_{2}(+\infty, t) & =P_{0} \\
T_{1}\left(r_{0}, t\right) & =T_{e} \\
T_{2}(+\infty, t) & =T_{0}
\end{aligned}
$$

The initial conditions are

$$
\begin{gathered}
P_{1}(r, 0)=P_{2}(r, 0)=P_{0} \\
T_{1}(r, 0)=T_{2}(r, 0)=T_{0}
\end{gathered}
$$

The continuity conditions at the decomposition front are

$$
\begin{gathered}
P_{1}\left(r_{c}, t\right)=P_{2}\left(r_{c}, t\right)=P_{\mathrm{D}}(t) \\
T_{1}\left(r_{c}, t\right)=T_{2}\left(r_{c}, t\right)=T_{\mathrm{D}}(t)
\end{gathered}
$$

Up to now, the problem to determine the pressure and temperature fields is, in mathematical, reduced into solving the mixed boundary value problem (1)-(15), for the partial differential equations in the two zones with moving interface.

\section{SEMI-ANALYTIC SOLUTION}

\subsection{The solution of pressure field}

Introduce the Boltzmann-transformation [12]:

$$
u=\frac{r^{2}}{4 \alpha_{1} t}
$$

and denote

$$
u_{c}(t)=\left.u\right|_{r=r_{c}(t)}=\frac{r_{c}(t)^{2}}{4 \alpha_{1} t}, \quad u_{e}(t)=\left.u\right|_{r=r_{0}}=\frac{r_{0}^{2}}{4 \alpha_{1} t}
$$


Thus, the pressures $P_{1}$ and $P_{2}$ are thought to be functions of new variable $u$. The partial differential equations (1)-(2) can be converted into

$$
\begin{array}{r}
u \frac{\mathrm{d}^{2} P_{1}}{\mathrm{~d} u^{2}}+(1+u) \frac{\mathrm{d} P_{1}}{\mathrm{~d} u}=0 \\
u \frac{\mathrm{d}^{2} P_{2}}{\mathrm{~d} u^{2}}+(1+N u) \frac{\mathrm{d} P_{2}}{\mathrm{~d} u}=0
\end{array}
$$

where the constant $N=\alpha_{1} / \alpha_{2}$.

The boundary conditions (8)-(9), the initial condition (12), and the continuity condition (14) can be written as

$$
\begin{aligned}
P_{1}\left(u_{e}\right) & =P_{e} \\
P_{1}\left(u_{c}\right) & =P_{2}\left(u_{c}\right)=P_{\mathrm{D}} \\
\lim _{u \rightarrow \infty} P_{1}(u) & =\lim _{u \rightarrow \infty} P_{2}(u)=P_{0}
\end{aligned}
$$

Integrating ordinary differential equations (18) and (19) yields

$$
\begin{aligned}
& P_{1}=C_{11} \int_{u_{e}}^{u} \frac{e^{-s}}{s} \mathrm{~d} s+C_{12} \\
& P_{2}=-C_{21} \int_{u}^{+\infty} \frac{e^{-N s}}{s} \mathrm{~d} s+C_{22}
\end{aligned}
$$

Using the boundary conditions (20)-(22), we can determine the integral constants $C_{k l}(k, l=1,2)$. Therefore, the semi-analytic solutions for the pressure field are given by

$$
\begin{aligned}
& P_{1}(u)=P_{e}+\left(P_{\mathrm{D}}-P_{e}\right) \frac{\int_{u_{e}}^{u} e^{-s} / s \mathrm{~d} s}{\int_{u_{e}}^{u_{c}} e^{-s} / s \mathrm{~d} s} \\
& P_{2}(u)=P_{0}-\left(P_{0}-P_{\mathrm{D}}\right) \frac{\int_{u}^{+\infty} e^{-N s} / s \mathrm{~d} s}{\int_{u_{c}}^{+\infty} e^{-N s} / s \mathrm{~d} s}
\end{aligned}
$$

\subsection{The solution for temperature field}

Using Boltzmann-transformation (16) again and substituting (25)-(26) into equations (3)-(4) lead to

$$
\begin{array}{r}
\left(u+C_{11} \frac{c_{\mathrm{w}} k_{1}}{c_{1} \mu \alpha_{1}} e^{-u}\right) \frac{\mathrm{d} T_{1}}{\mathrm{~d} u}-C_{11}^{2} \frac{c_{\mathrm{w}} k_{1} \delta}{c_{1} \mu \alpha_{1}} \frac{e^{-2 u}}{u}-C_{11} \frac{\eta \phi_{1} c_{\mathrm{w}}}{c_{1}} e^{-u}=0 \\
\left(u+C_{21} \frac{c_{\mathrm{w}} k_{2}}{c_{2} \mu \alpha_{1}} e^{-N u}\right) \frac{\mathrm{d} T_{2}}{\mathrm{~d} u}-C_{21}^{2} \frac{c_{\mathrm{w}} k_{2} \delta}{c_{2} \mu \alpha_{1}} \frac{e^{-2 N u}}{u}-C_{21} \frac{\eta \phi_{2} c_{\mathrm{w}}}{c_{2}} e^{-N u}=0
\end{array}
$$


The boundary conditions (10) and (11), the initial condition (13), the continuity condition (15) can be written as

$$
\begin{aligned}
T_{1}\left(u_{e}\right) & =T_{e} \\
T_{1}\left(u_{c}\right) & =T_{2}\left(u_{c}\right)=T_{\mathrm{D}} \\
T_{2}(+\infty) & =T_{0}
\end{aligned}
$$

Solving the boundary value problem of ordinary differential equations (27)-(31), we have the semi-analytic solutions for temperature field:

$$
\begin{aligned}
& T_{1}(u)=T_{e}+\left(T_{\mathrm{D}}-T_{e}\right) \frac{\int_{u_{e}}^{u} G_{1}(s) \mathrm{d} s}{\int_{u_{e}}^{u_{c}} G_{1}(s) \mathrm{d} s} \\
& T_{2}(u)=T_{0}+\left(T_{\mathrm{D}}-T_{0}\right) \frac{\int_{u}^{+\infty} G_{2}(s) \mathrm{d} s}{\int_{u_{c}}^{+\infty} G_{2}(s) \mathrm{d} s}
\end{aligned}
$$

where

$$
\begin{aligned}
& G_{1}(u)=\left[\frac{k_{1} \delta}{\mu \alpha_{1}} \frac{\left(P_{\mathrm{D}}-P_{e}\right)}{\int_{u_{e}}^{u_{c}} e^{-s} / s \mathrm{~d} s} \frac{e^{-u}}{u}+\eta \phi_{1}\right]\left[\frac{c_{1} \int_{u_{e}}^{u_{c}} \mathrm{e}^{-s} / s \mathrm{~d} s}{c_{\mathrm{W}}\left(P_{\mathrm{D}}-P_{e}\right)} u e^{u}+\frac{k_{1}}{\mu \alpha_{1}}\right]^{-1}, \\
& G_{2}(u)=\left[\frac{k_{2} \delta}{\mu \alpha_{1}} \frac{P_{0}-P_{\mathrm{D}}}{\int_{u_{c}}^{+\infty} e^{-N s} / s \mathrm{~d} s} \frac{e^{-N u}}{u}+\eta \phi_{2}\right]\left[\frac{c_{2} \int_{u_{c}}^{+\infty} e^{-N s} / s \mathrm{~d} s}{c_{\mathrm{w}}\left(P_{0}-P_{\mathrm{D}}\right)} u e^{N u}+\frac{k_{2}}{\mu \alpha_{1}}\right]^{-1}
\end{aligned}
$$

\subsection{Determination of unknown functions}

The above solutions (25)-(26) and (32)-(33) are not fully determined, as $P_{\mathrm{D}}(t)$ and $T_{\mathrm{D}}(t)$ are unknown functions. This section presents the process of determination of the unknown functions $P_{\mathrm{D}}(t)$ and $T_{\mathrm{D}}(t)$. The Boltzmann-transformation (16) is used at the decomposition front $u=u_{c}(t)$ $\left(r=r_{c}(t)\right)$, then the mass and the energy balance equations (6) and (7) become

$$
\begin{array}{r}
\left.\left(\chi_{1} \frac{\mathrm{d} P_{1}}{\mathrm{~d} u}-\chi_{2} \frac{\mathrm{d} P_{2}}{\mathrm{~d} u}\right)\right|_{u=u_{c}} \frac{r_{c}(t)}{2 \alpha_{1} t}=-\frac{\mathrm{d} r_{c}}{\mathrm{~d} t} \\
\left.\left(\xi_{1} T_{\mathrm{D}} \frac{\mathrm{d} P_{1}}{\mathrm{~d} u}-\xi_{2} T_{\mathrm{D}} \frac{\mathrm{d} P_{2}}{\mathrm{~d} u}\right)\right|_{u=u_{c}} \frac{r_{c}(t)}{2 \alpha_{1} t}=-\frac{\mathrm{d} r_{c}}{\mathrm{~d} t}
\end{array}
$$

Comparing the two sides in equations (34) and (35) yields

$$
\left.\left(\chi_{1}-\xi_{1} T_{\mathrm{D}}\right) \frac{\mathrm{d} P_{1}}{\mathrm{~d} u}\right|_{u=u_{c}}=\left.\left(\chi_{2}-\xi_{2} T_{\mathrm{D}}\right) \frac{\mathrm{d} P_{2}}{\mathrm{~d} u}\right|_{u=u_{c}}
$$


Substituting the solutions (25)-(26) and (17) into (34) and (36), respectively, we have

$$
\begin{aligned}
\frac{\mathrm{d} r_{c}}{\mathrm{~d} t}= & -\frac{2 \chi_{1} \alpha_{1} t\left(P_{\mathrm{D}}\left(T_{\mathrm{D}}\right)-P_{e}\right) e^{-r_{c}^{2} / 4 \alpha_{1} t}}{r_{c}\left[E i\left(-\frac{r_{c}^{2}}{4 \alpha_{1} t}\right)-E i\left(-\frac{r_{0}^{2}}{4 \alpha_{1} t}\right)\right]} \\
& +\frac{2 \chi_{2} \alpha_{1} t\left(P_{\mathrm{D}}\left(T_{\mathrm{D}}\right)-P_{0}\right) e^{-N r_{c}^{2} / 4 \alpha_{1} t}}{r_{c} E i\left(-\frac{N r_{c}^{2}}{4 \alpha_{1} t}\right)} \\
\frac{\left(\chi_{1}-\xi_{1} T_{\mathrm{D}}\right)\left(P_{\mathrm{D}}\left(T_{\mathrm{D}}\right)-P_{e}\right)}{\left(\chi_{2}-\xi_{2} T_{\mathrm{D}}\right)\left(P_{\mathrm{D}}\left(T_{\mathrm{D}}\right)-P_{0}\right)}= & \frac{E i\left(-\frac{r_{c}^{2}}{4 \alpha_{1} t}\right)-E i\left(-\frac{r_{0}^{2}}{4 \alpha_{1} t}\right)}{E i\left(-\frac{N r_{c}^{2}}{4 \alpha_{1} t}\right)} e^{(N-1) \frac{r_{c}^{2}}{4 \alpha_{1} t}}
\end{aligned}
$$

in which the unknown $P_{\mathrm{D}}$ can be expressed from the phase equilibrium equation (5) as

$$
P_{\mathrm{D}}=P_{\mathrm{D}}\left(T_{\mathrm{D}}\right)=10^{a_{0}\left(T_{\mathrm{D}}-T_{0}\right)+b_{0}\left(T_{\mathrm{D}}-T_{0}\right)^{2}+c_{0}}
$$

where Ei is the exponential integral function defined as

$$
\operatorname{Ei}(-u)=-\int_{u}^{\infty} \frac{e^{-x}}{x} \mathrm{~d} x \quad(0<u<+\infty)
$$

Then, introduce new variable

$$
y(t)=r_{c}^{2}(t) / 4 \alpha_{1}
$$

From equation (37) and the initial condition $r_{c}(0)=r_{0}$, we can obtain

$$
\frac{\mathrm{d} y}{\mathrm{~d} t}=f_{1}\left(t, y, T_{\mathrm{D}}\right), \quad y(0)=y_{0}
$$

where the function

$$
f_{1}\left(t, y, T_{\mathrm{D}}\right)=-\frac{\chi_{1}\left(P_{\mathrm{D}}\left(T_{\mathrm{D}}\right)-P_{e}\right) \mathrm{e}^{-y / t}}{\alpha_{1}\left[E i(-y / t)-E i\left(-y_{0} / t\right)\right]}+\frac{\chi_{2}\left(P_{\mathrm{D}}\left(T_{\mathrm{D}}\right)-P_{0}\right) \mathrm{e}^{-N y / t}}{\alpha_{1} E i(-N y / t)}
$$

and the initial value $y_{0}=r_{0}^{2} /\left(4 \alpha_{1}\right)$.

From the condition (10) and (15), we know $T_{\mathrm{D}}(0)=T_{1}\left(r_{0}, 0\right)=T_{e}$. Then, giving derivation of equation (38) yields

$$
\frac{\mathrm{d} T_{\mathrm{D}}}{\mathrm{d} t}=f_{2}\left(t, y, T_{\mathrm{D}}\right), \quad T_{\mathrm{D}}(0)=T_{e}
$$


where the function

$$
\begin{aligned}
f_{2}\left(t, y, T_{\mathrm{D}}\right) & =\frac{F_{1}\left(T_{\mathrm{D}}\right)}{F_{2}(t, y)} f_{1}\left(t, y, T_{\mathrm{D}}\right) \\
F_{1}\left(T_{\mathrm{D}}\right) & =\frac{\left(\chi_{1} \xi_{2}-\chi_{2} \xi_{1}\right)\left(P_{\mathrm{D}}-P_{e}\right)}{\left(\chi_{2}-\xi_{2} T_{\mathrm{D}}\right)^{2}\left(P_{\mathrm{D}}-P_{0}\right)}+\frac{\left(\chi_{1}-\xi_{1} T_{\mathrm{D}}\right)\left(P_{e}-P_{0}\right)}{\left(\chi_{2}-\xi_{2} T_{\mathrm{D}}\right)\left(P_{\mathrm{D}}-P_{0}\right)^{2}} P_{\mathrm{D}} \ln 10\left(a_{0}+2 b_{0}\left(T_{\mathrm{D}}-T_{0}\right)\right) \\
F_{2}(t, y) & =\frac{t \mathrm{e}^{-N y / t}}{y E i\left(-N y_{0} / t\right)}+\frac{\left[E i(-y / t)-E i\left(-y_{0} / t\right)\right](1-N) \mathrm{e}^{-(1-N) y / t}}{t E i\left(-N y_{0} / t\right)}
\end{aligned}
$$

Up to now, determination of unknown functions is reduced to solve the initial value problem of ordinary differential equations (41) and (42).

\subsection{Numerical solving procedure}

The procedure of numerical calculation are given as below:

(1) Give initial values $y(0)=y_{0}, T_{\mathrm{D}}(0)=T_{e},(41)$ and (42) can be solved by the Runge-Kutta method, then $y(t)$ and $T_{\mathrm{D}}(t)$ were determined;

(2) The decomposition pressure $P_{\mathrm{D}}$ and the location of decomposition front $r_{c}$ can be solved by (39) and (40);

(3) After the value above-mentioned has been obtained, any given time $t$ and distance $r$, we can get the distribution of the pressure by (25)-(26) and the distribution of temperature by (32)-(33).

\subsection{The gas production rate}

The gas production rate can be calculated by the following formation:

$$
\frac{\mathrm{d} Q}{\mathrm{~d} t}=q \frac{\varepsilon \rho}{\rho_{h}} \cdot 2 \pi h r_{c}(t) \frac{\mathrm{d} r_{c}(t)}{\mathrm{d} t}
$$

Table I. Values of parameter.

\begin{tabular}{lcclcc}
\hline Parameter & & Value & \multicolumn{1}{c}{ Parameter } & Value \\
\hline Water volume heat capacity & $c_{\mathrm{W}}$ & $4200 \mathrm{~J} / \mathrm{kg}^{\circ} \mathrm{C}$ & Density of water & $\rho_{w}$ & $1000 \mathrm{~kg} / \mathrm{m}^{3}$ \\
Sediment volume heat capacity & $c$, & $1500 \mathrm{~J} / \mathrm{kg}^{\circ} \mathrm{C}$ & Density of sediment & $\rho$ & $2000 \mathrm{~kg} / \mathrm{m}^{3}$ \\
Gas produced after $1 \mathrm{~m}^{3}$ & & & & & \\
hydrate decompose & $q$ & $164 \mathrm{~m}^{3}$ & Sediment Poisson's ratio & $v_{1}$ & 0.4 \\
Throttling coefficient & $\delta$ & $8 \times 10^{-7} \mathrm{~K} / \mathrm{Pa}$ & Sediment Poisson's ratio & $v_{2}$ & 0.3 \\
Adiabatic coefficient & $\eta$ & $8 \times 10^{-7} \mathrm{~K} / \mathrm{Pa}$ & Sediment Young's modulus & $E_{1}$ & $10 \mathrm{MPa}$ \\
Porosity of the sediment & $\phi_{1}$ & 0.4 & Sediment Young's modulus & $E_{2}$ & $50 \mathrm{MPa}$ \\
Porosity of the sediment & $\phi_{2}$ & 0.28 & Viscosity of water & $\mu$ & $1.3 \times 10^{-6} \mathrm{ps}$ \\
Mass fraction of hydrate & $\varepsilon$ & $5.4 \%$ & Latent heat of hydrate & $L$ & $4.3 \times 10^{5} \mathrm{~J} / \mathrm{kg}$ \\
\hline
\end{tabular}




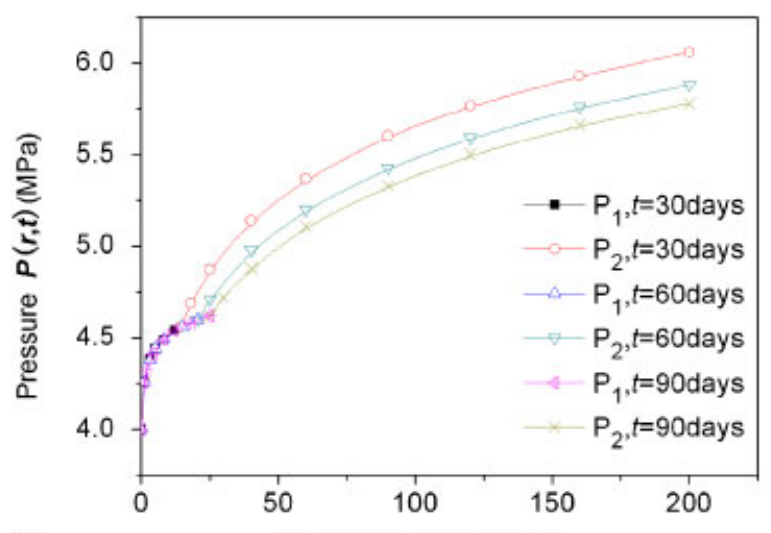

(a)

Radial coordinate $r(m)$

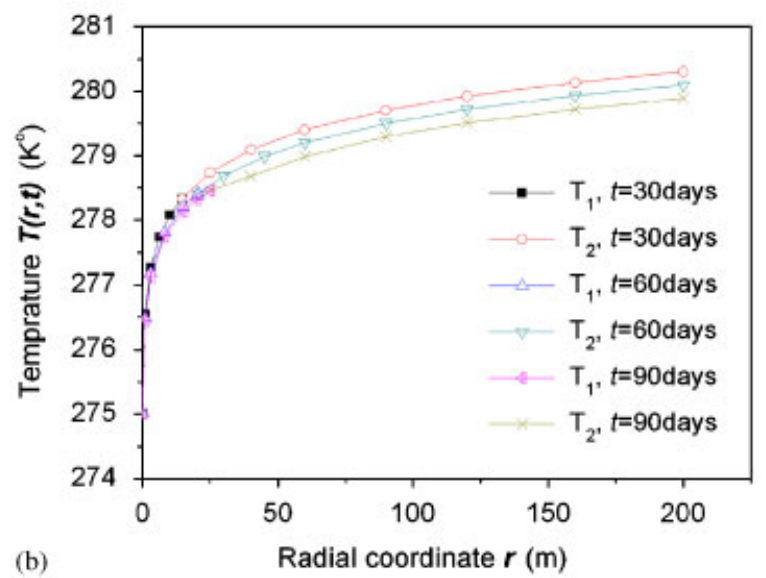

Figure 2. (a) The pressure profiles at 30,60, 90 days for a well pressure of $4 \mathrm{MPa}$ and a well temperature of $275 \mathrm{~K}\left(k_{1}=14 \mathrm{md}, k_{2}=4 \mathrm{md}\right)$ and (b) the temperature profiles at 30, 60, 90 days for a well pressure of $4 \mathrm{MPa}$ and a well temperature of $275 \mathrm{~K}\left(k_{1}=14 \mathrm{md}, k_{2}=4 \mathrm{md}\right)$.

where $Q$ is the volume of gas produced, $q$ is gas produced by $1 \mathrm{~m}^{3}$ gas hydrate, $h$ is the thickness of hydrate layer [13]. Using (40) and (41), we have

$$
\frac{\mathrm{d} Q}{\mathrm{~d} t}=q \frac{\pi h \varepsilon \rho}{2 \alpha_{1} \rho_{h}} f_{1}\left(t, y(t), T_{\mathrm{D}}(t)\right)
$$

\section{RESULTS}

Numerical results are presented in this section. The pressure and temperature profiles in reservoir for different times, the decomposition front location profiles over time under different conditions are obtained. The influence of permeability and the well pressure on the speed of decomposition front is discussed below. It is assumed that the initial pressure of the reservoir is $8 \mathrm{MPa}$ and 

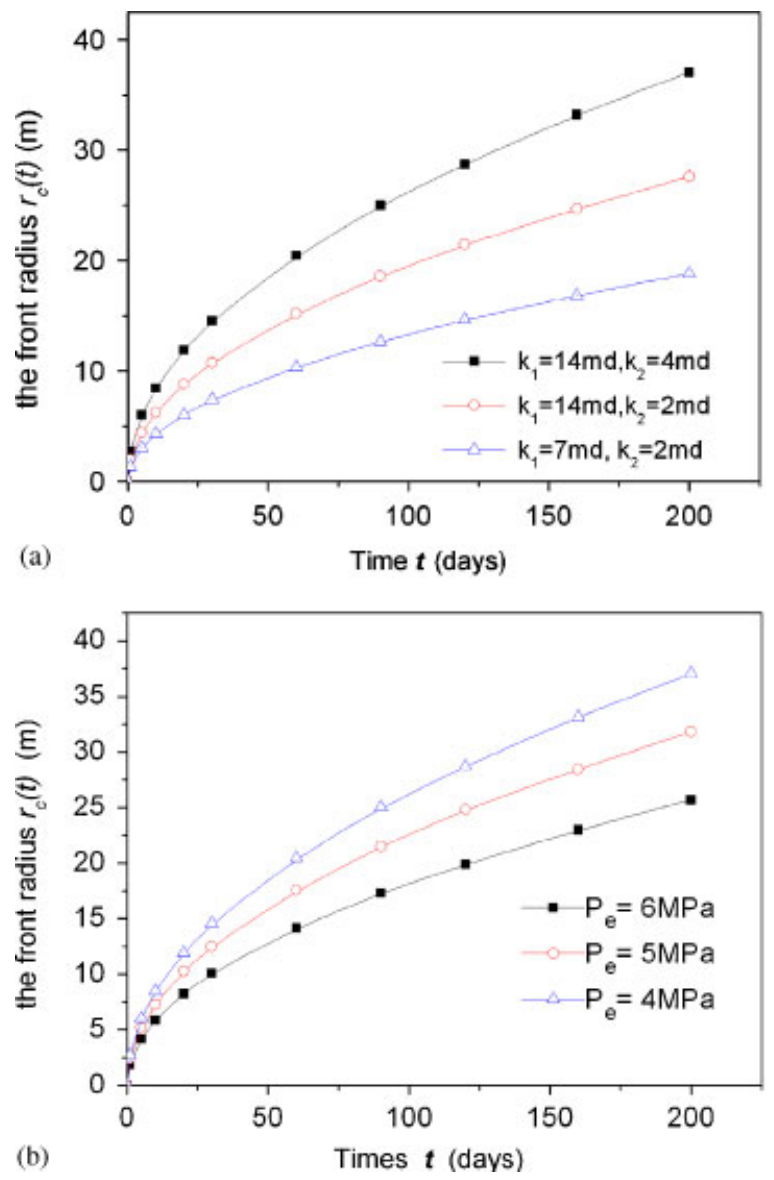

Figure 3. (a) The curves of decomposition front radius $r_{c}(t)$ for various permeabilities $k_{1}$ and $k_{2}\left(P_{e}=4 \mathrm{MPa}\right)$ and $(\mathrm{b})$ the relationship between the location of the decomposition front and time at different permeability $\left(k_{1}=14 \mathrm{md}, k_{2}=4 \mathrm{md}\right)$.

the initial temperature of the reservoir is $283 \mathrm{~K}$. As an example of numerical computation, the parameters are given as below (Table I).

Figure 2 shows the pressure and temperature profiles for different times under the conditions that $K_{1}=14 \mathrm{md}, K_{2}=4 \mathrm{md}, P_{e}=4 \mathrm{MPa}, T_{e}=275 \mathrm{~K}$. As shown in Figure 2, the pressure and the temperature decrease from the decomposition front to the well in zone 1, and the gradient of pressure and temperature is largest near the well. The pressure and the temperature change sharply near the decomposition front in the hydrate zone, and the gradient of pressure and temperature is low in the distance far from the decomposition front.

For different permeabilities of sediment and well pressure, the time variation of decomposition front location is presented in Figure 3. As shown in Figure 3(a), the well pressure and temperature are kept $4 \mathrm{MPa}$ and $275 \mathrm{~K}$, the decomposition moves slower with time, if the permeability in zone 1 or 2 be improved, the distance of decomposition front increases evidently. Figure 3(b) shows the influence of the well pressure on the distance of decomposition. For a well pressure of $6 \mathrm{MPa}$, 

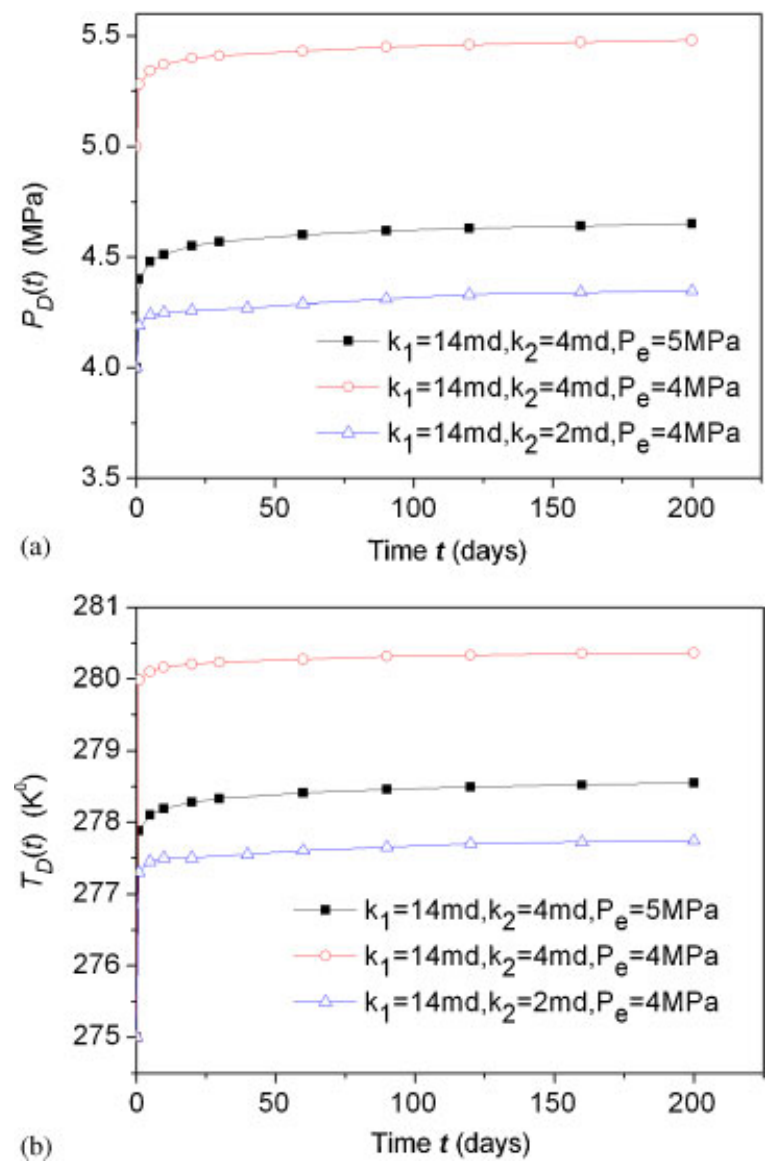

Figure 4. (a) The relationship between the pressure $P_{\mathrm{D}}$ on the decomposition front and time and (b) the relationship between the temperature $T_{\mathrm{D}}$ on the decomposition front and time.

the decomposition front is about $22 \mathrm{~m}$ to the well center after 200 days, and for a well pressure of $4 \mathrm{MPa}$, the distance is about $35 \mathrm{~m}$ under the same conditions. This shows that the well pressure has much influence on the speed of decomposition front, the decomposition front moves faster when the well pressure decreases.

Figure 4 compares the variations of the pressure and the temperature at the decomposition front with time under different conditions. As can be seen from Figure 6, the pressure and the temperature at the decomposition front change sharply at initial time, and become nearly a constant after a short time.

The changes of pressure $P_{\mathrm{D}}$ and temperature $T_{\mathrm{D}}$ at the decomposition front as the well pressure changes under different conditions are compared in Figure 5. As can be seen from Figure 5(a), $P_{\mathrm{D}}$ increases linearly almost as the well pressure increases. As $P_{\mathrm{D}}$ is determined by the mass and heat balance equation at the decomposition front, the well pressure has effect on the pressure gradient at the decomposition front, subsequently it has an evident influence on the decomposition pressure $P_{\mathrm{D}}$. As there is relationship between $T_{\mathrm{D}}$ and $P_{\mathrm{D}}$ shown in equation $(5), T_{\mathrm{D}}$ has similar variation rule shown in Figure 5(b). 

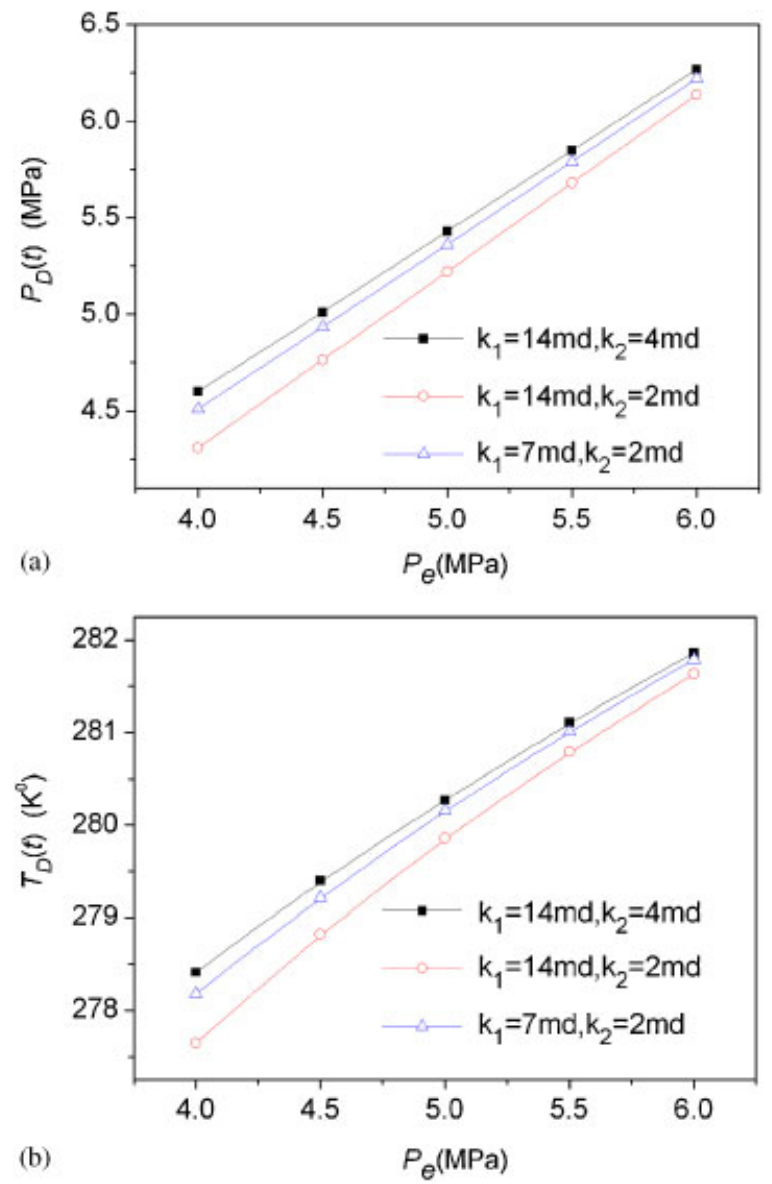

Figure 5. (a) The relationship between the pressure $P_{\mathrm{D}}$ on the decomposition front and well pressure and (b) the relationship between the pressure $T_{\mathrm{D}}$ on the decomposition front and well pressure.

Figure 6 presents gas production rate changes with well pressure and permeability of sediment. As shown in Figure 6(a), dropping well pressure can increase gas production rate obviously. As shown from Figure 6(b), the permeability of sediment has strong influence on the gas production rate. If the permeability of sediment increases, the gas production rate of gas increases evidently. It also shows that the gas production rate becomes slower in initial period, and becomes a constant after a short time.

\section{CONCLUSIONS}

The Stephen problem during gas production from gas hydrates was solved by the method Boltzmann-transformation. The semi-analytic solutions for pressure and temperature distribution were obtained in which the mass and energy balance equations at the decomposition front were 

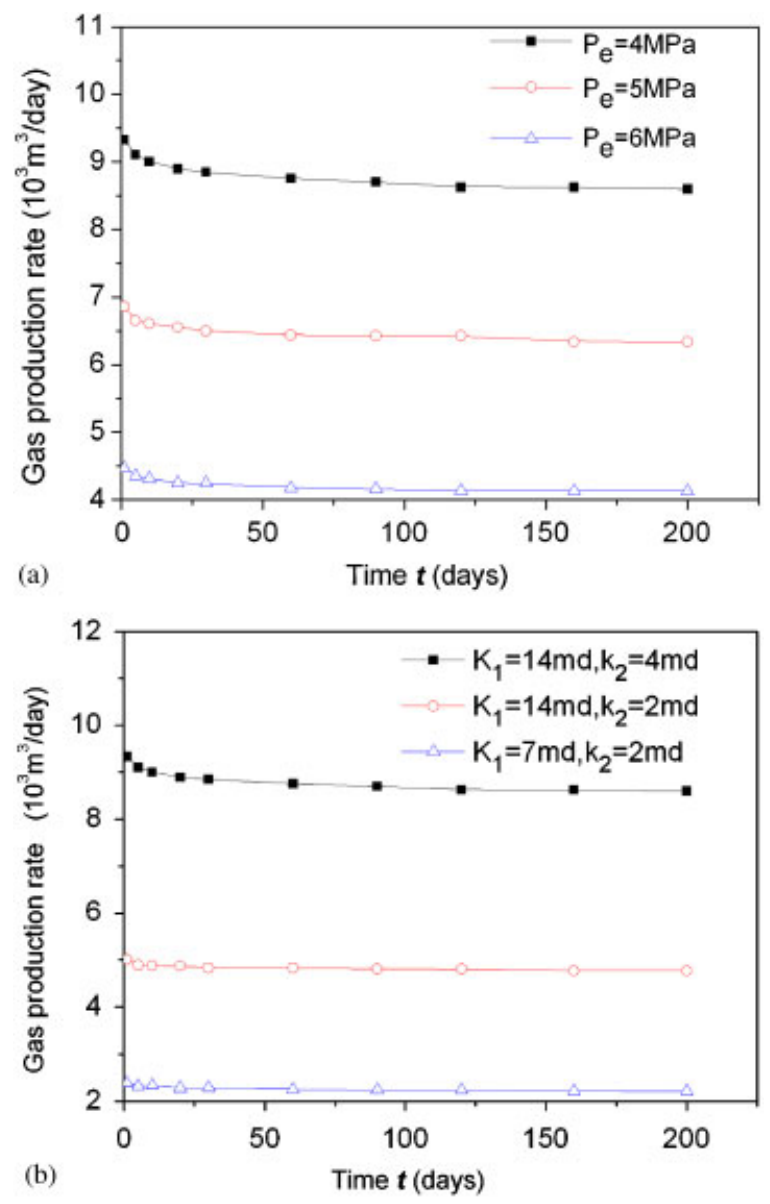

Figure 6. (a) Gas production rate changes with time at different well pressure and (b) gas production rate changes with time at different permeability.

considered, and the latent heat of gas hydrate was reckoned in. The numerical results could predict the volume of gas produced and provide some possible routes for raising gas production rate in the actual production. The following conclusions were drawn from the solutions:

(1) As the energy balance equation on the decomposition front joins to the mathematical modeling, the effect of latent heat of gas hydrate on the front temperature $T_{\mathrm{D}}$ is considered. The obtained semi-analytic solutions indicate sharp changes of the front temperature $T_{\mathrm{D}}$ and the pressure $P_{\mathrm{D}}$ in initial time. With time, the front temperature $T_{\mathrm{D}}$ and the pressure $P_{\mathrm{D}}$ reach asymptotically a constant.

(2) The influence of the well pressure and the sediment permeability on the gas production rate is evident. In the case of constant well pressure, the gas production rate is close to a constant after a short initial time. Decreasing well pressure could increase the gas production rate. 


\section{REFERENCES}

1. Holder GD, Angert PF, Godbole SP. Simulation of gas production from a reservoir containing both gas hydrates and free natural gas. SPE 11005, 57th Annual Conference, New Orleans, September 1982; 26-29.

2. Burshears M, O’Brien TJ, Malone RD. A multi-phase, multi-dimensional, variable composition simulation of gas production from a conventional gas reservoir in contact with hydrates. SPE 15246, Unconventional Gas Technology Symposium, Louisville, KY, May 1986; 18-21.

3. Yousif MH, Li PM, Selim MS, Sloan ED. Depressurization of natural gas hydrates in berea sandstone cores. Journal of Inclusion Phenomena and Molecular Recognition in Chemistry 1990; 8:71-88.

4. Yousif MH, Abass HH, Selim MS, Sloan ED. Experimental and theoretical investigation of methane-gas-hydrate dissociation in porous media. SPE Reservoir Engineering 1991; 6:69-76.

5. Durgut I, Parlaktuna M. A numerical method for the gas production process in gas hydrate reservoirs. Second International Conference on Natural Gas Hydrates, Toulouse, France, 1996.

6. Makogon YF. Hydrates of Hydrocarbons. Penn Well: Tulsa, OK, 1997.

7. Sloan Jr ED. Clathrate Hydrates of Natural Gases (2nd edn). Marcel Dekker: New York, 1998.

8. Tsypkin G. Mathematical Models of Gas Hydrates Dissociation in Porous Media. New York Academy of Sciences: New York, 2000; 912-928.

9. Ji C, Ahmadi G, Smith DH. Natural gas production from hydrate decomposition by depressurization. Chemical Engineering Science 2001; 56(20):5801-5814.

10. Ahmadi G, Ji C, Smith DH. Numerical solution for natural gas production from methane hydrate dissociation. Journal of Petroleum Science and Engineering 2004; 41(4):269-285.

11. Ahmadi G, Ji C, Smith DH. Production of natural gas from methane hydrate by a constant down-hole pressure well. Energy Conversion and Management 2007; 48(7):2053-2068.

12. Janz M. Moisture diffusivities evaluated at high moisture levels from a series of water absorption tests. Materials and Structures 2002; 35:141-148.

13. George J, Moridis E. Dendy Sloan. Gas production potential of disperse low-saturation hydrate accumulations in oceanic sediments. Energy Conversion and Management 2007; 48:1834-1849. 